\title{
Case Report \\ Idiopathic Granulomatous Mastitis in Pregnancy: A Case Report
}

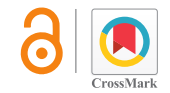

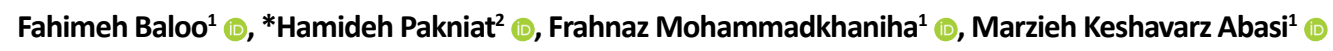

1. Kosar Medical Educational Center, Qazvin University of Medical Science, Qazvin, Iran.

2. Department of Obstetrics and Gynecology, School of Medicine, Qazvin University of Medical Sciences, Qazvin, Iran.

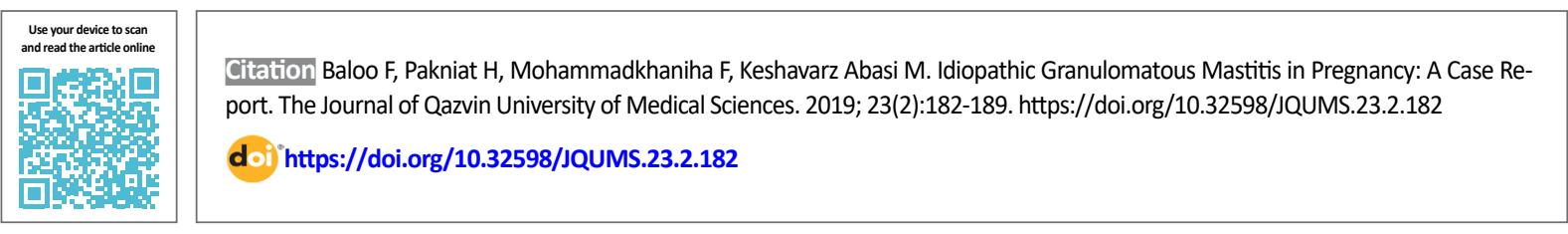

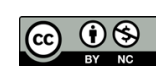

Received: 4 Oct 2018

Accepted: 27 Jan 2019

Available Online: 01 Apr 2019

Keywords:

Granulomatous

mastitis, Case report, Pregnancy

\section{ABSTRACT}

Idiopathic granulomatous mastitis is an inflammatory disease with unknown etiology. The clinical signs and mammograms of the disease can mimic breast cancer. The present study reports a case of idiopathic granulomatous mastitis in a pregnant woman. A 26-year-old woman, who was 24 weeks pregnant, felt a mass on her left breast. Biopsy of the mass revealed no evidence of malignancy. For treatment, intravenous and oral prednisolone tablets were prescribed during the pregnancy, which was terminated without a problem under normal care.

\section{Extended Abstract}

\section{Introduction}

I

diopathic Granulomatous Mastitis (IGM) is a benign inflammatory breast disease, which usually occurs in young women of childbearing age, mothers who have given birth, or the breast-feeding ones [1]. The etiology of the disease is unknown, but studies have associated it to autoimmune diseases, response to trauma, and hormonal or metabolic disorders [2-4]. It is also assumed to be related to infectious diseases such as sarcoidosis, tuberculosis, actinomycosis, blastomycosis, filariasis, and Corynebacterium spp [5-9].
IGM's most common symptoms include unilateral painful swollen breast mass with skin redness or ulcer, nipple discharge, nipple retr action, or feeling of fullness under the arm $[2,10]$. In mammography, IGM is shown as asymmetric focal density with irregular borders, irregular mass, or multiple bilateral nodules. In ultrasonography, hypoechogenic lesions are observed with abscess cavities. Other methods for diagnosis of IGM are MRI and Doppler ultrasonography [11].

Currently, no definitive cure exists for IGM. Some of the therapeutic methods are antibiotic therapy, corticosteroid therapy, and the use of immunosuppressive agents as well as aggressive approaches such as surgery and abscess drainage [12]. IGM is a rare and benign breast disease in pregnant women [13]. Since the clinical appearance of

\section{. \\ * Corresponding Author: \\ Hamideh Pakniat}

Address: Department of Obstetrics and Gynecology, School of Medicine, Qazvin University of Medical Sciences, Qazvin, Iran.

Tel: +98 (28) 33236374

E-Mail: hpalniat@qums.ac.ir 
IGM is similar to that of breast cancer, and there are few studies in pregnant women in this area, we present a case report of IGM in a pregnant woman.

\section{Case Report}

The patient was a 26 years old pregnant woman, with second pregnancy and history of previous cesarean section and gestational age of about 24 weeks referred for pregnancy care following the feeling of a mass in her left breast. In the examination, a firm mass of $40 \times 40 \mathrm{~mm}$ (Figure 1) was palpated, and ultrasonography images showed multiple collections of mass sized $85 \times 35 \mathrm{~mm}$, and increased echogenicity. The patient underwent coreneedle biopsy for the diagnosis, and sampling was conducted at 1 and 3 PM in two areas.

By observing neutrophils and histiocytes accumulation around a space, the IGM was diagnosed. Then, treatment with prednisolone at a dosage of $25 \mathrm{mg}$ per day along with glycemic control was started. The patient underwent prenatal care during pregnancy, and the pregnancy passed without any problem. At 38 weeks and 4 days of gestation, pregnancy was terminated by cesarean section due to uterine rupture and having a previous cesarean section. The newborn was physically examined, and no problem was observed. After the cesarean section, 50mg hydrocortisone was administered intravenously three times a day in the first two days, and then the dose was tapered to $25 \mathrm{mg}$ twice a day on the third day and finally once daily on the fourth day.On the fifth day (discharge time), surgical consultation was given again, and the patient was discharged with a prescription of prednisolone $5 \mathrm{mg}$ in the morning and 10mg at noon and night. Follow-up for IGM continued up to 3 weeks after discharge.

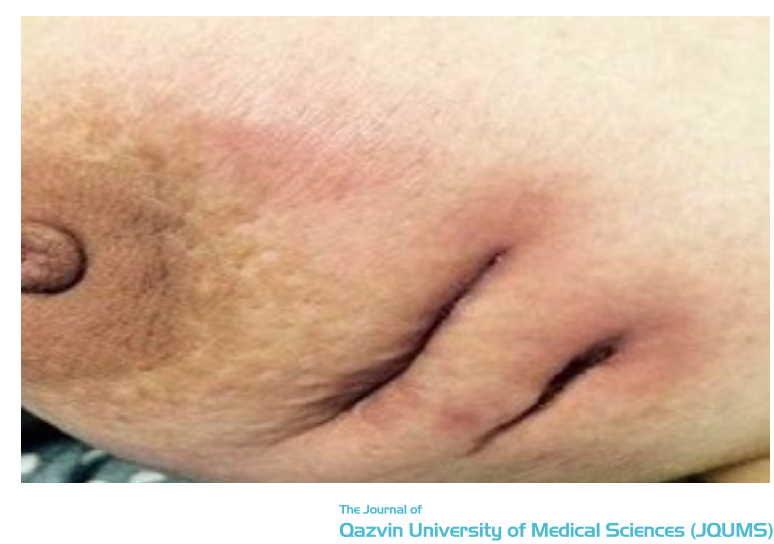

Figure 1. The left breast of the patient with IGM

\section{Discussion and Conclusion}

IGM is more common in women of childbearing age, but studies report the different ages in the patients, with one case reported in an 11-year-old girl [16]. Factors associated with this disease include the use of oral contraceptives, autoimmune diseases, and hyperprolactinemia, pregnancy, and lactation [6]. In a study conducted by Pourzand and Mohammadzadeh-Gharabaghi [14] on 38 patients with IGM, the most common clinical manifestations were abscess, erythema, edema, and inflammation. The ultrasound results showed a hypoechoic fibroglandular mass in the superior lateral quadrant or central region. Diagnostic tools for IGM include ultrasonography, mammography, contrastenhanced MRI, which are not specific.

Other methods are biopsy and fine-needle aspiration, which are often recommended as a diagnostic procedure $[11,17]$. For example, Pourzand and MohammadzadehGharabaghi, Kadivar et al. [16], and Mizrakli et al. used ultrasonography and fine-needle aspiration for the diagnosis of IGM. Its differential diagnoses include malignancy, infection, autoimmune diseases, and other granulomatous diseases such as tuberculosis and sarcoidosis [6-11, 14, 16, 17]. In the study of Kadivar et al. there was no sign of infection or trauma.

Over 50 percent of IGM cases can show symptoms similar to those of breast carcinoma $[16,17]$. Since the study of patients with these clinical manifestations needs to rule out other causes of mastitis and malignancy, the biopsy is essential for pathological examination. There is no uniformly accepted treatment for IGM, but surgery and long-term treatment with corticosteroids and immunosuppressant drugs are recommended. In pregnant women, due to the contraindication of some drugs and their adverse effects on the fetus, the definite diagnosis of the problem and its differentiation from other cases is necessary.

\section{Ethical Considerations}

\section{Compliance with ethical guidelines}

This study was performed by providing explanations to the patient and obtaining her consent to report the case as a research project and to preserve her confidentiality.

\section{Funding}

This research was extracted from the research project, funded by Qazvin Kosar Educational and Medical Center. 


\section{Authors' contributions}

Compiling information and participating in case report and conceptualization: Fahimeh Baloo; Introducing a sample, pursuing patient's care, monitoring and project management: Hemidah Pakniat; Collecting information and writing draft and editing references; Frahnaz Mohammadkhaniha; Searching and translating related articles and compiling information and writing the draft: Marzieh Keshavarz Abasi.

\section{Conflicts of interest}

The authors declared no conflict of interest.

Acknowledgements

The authors would like to thank the Clinical and Development Research Center and Research Committee of Kosar hospital, Qazvin University of Medical Sciences. 


\title{
ماستيت ترانولوماتوز ايديوياتيك در باردارى: تزارش مورد
}

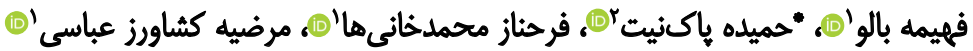

ا- ا-مركز آموزشى و درمانى كوثر، دانشكاه علوم يزشكى قزوين ، قزوزين، ايران

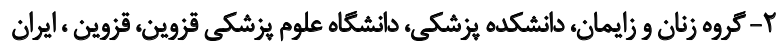

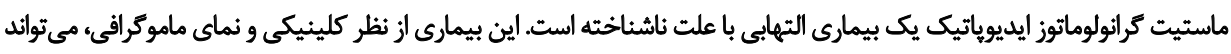

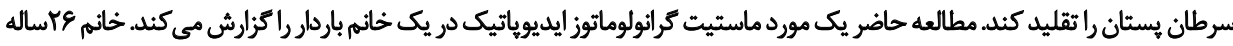

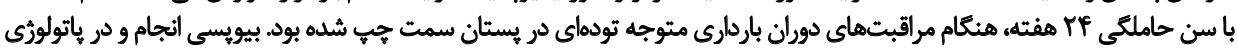

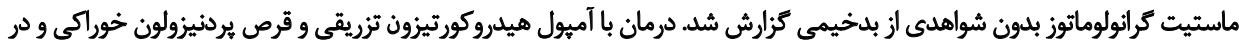

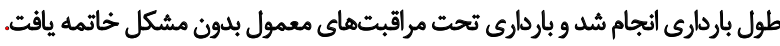

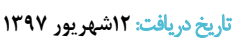

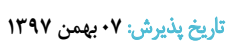

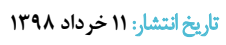 \\ كائلدوالزها: \\ ماستيت كرا انولوماتوزة \\ اليديوياتيك، باردارى
}

نماى ماموكرافى بيمارى مي تواند به صورت دانهي نانسيته فوكال

dates

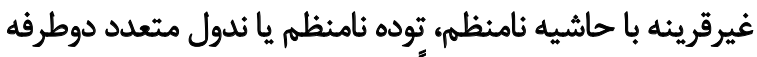

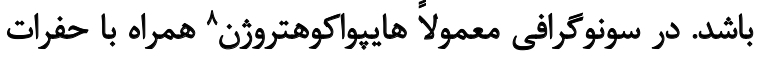

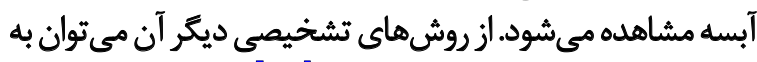

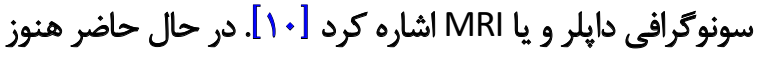

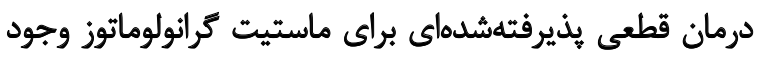

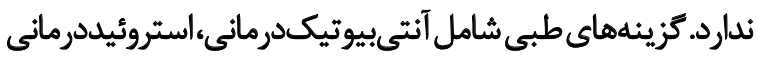

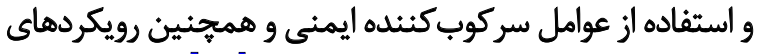

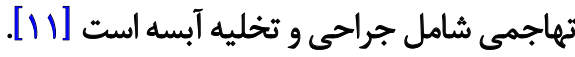

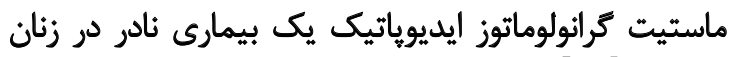

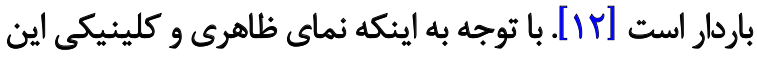

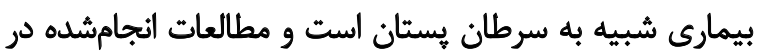

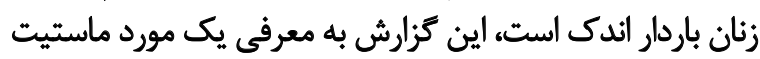
كرانولوماتوز ايديوياتيك در يك خانئ باني باردار مي بيردازد.

$$
\text { معرفي بيمار }
$$

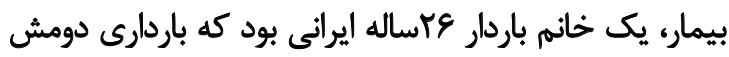

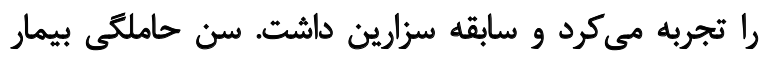

\section{Hypoechogenic}

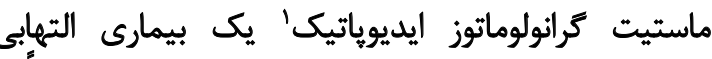

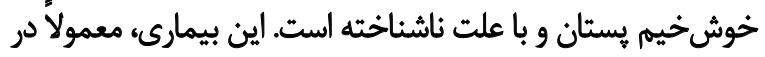

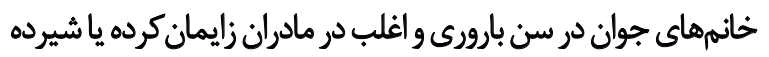

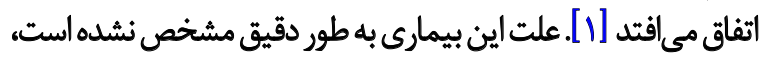

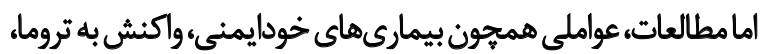

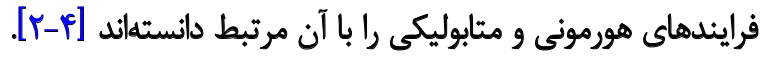

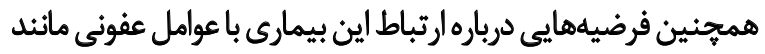

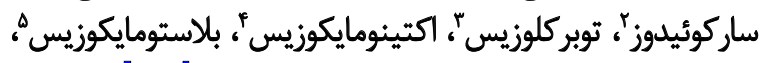

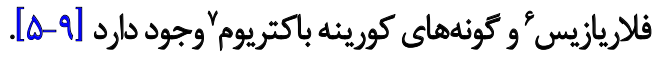

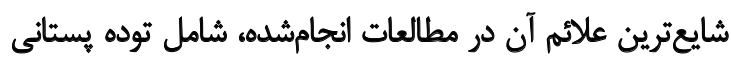

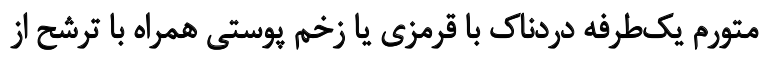

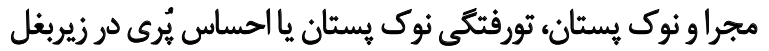

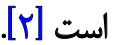

1. Idiopathic granulomatous mastits (IGM)

2. Sarcoidosis

3. Tuberculosis

4. Actinomycosis

5. Blastomycosis

6. Filariasis

7. Corynebacterium

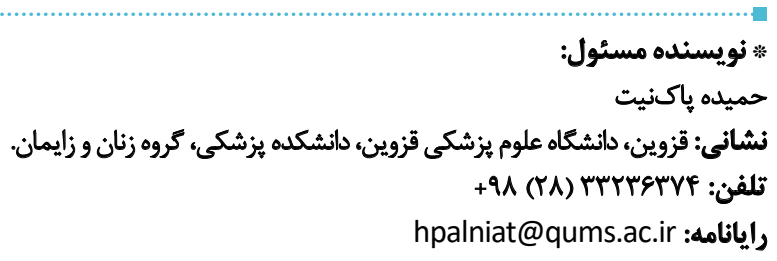


بيمار در طول باردارى تحت مراقبتهاى دقيق دوران باردارى

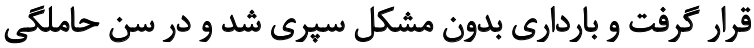

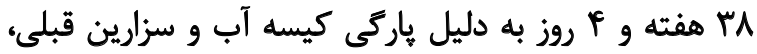

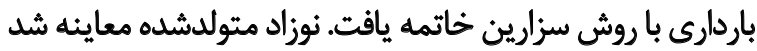
و مشكلى مشاهده نشد.

يس از انجام سزارين، طبق مشاوره جراحى كه در بخش بعد از

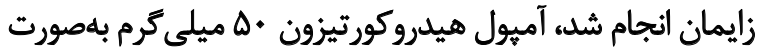

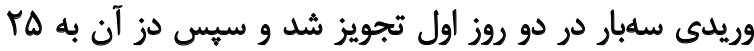

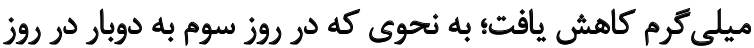

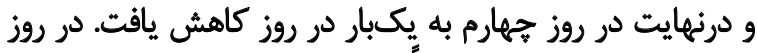

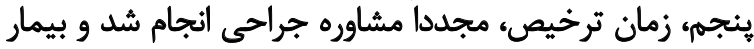

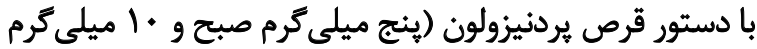

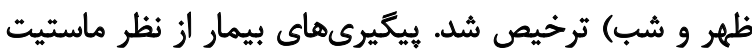

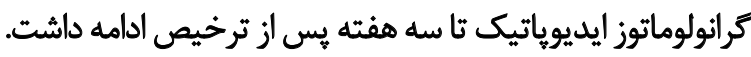

\section{بحث و نتيجنه كَيرى}

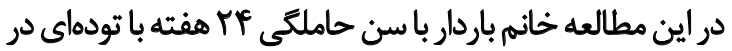

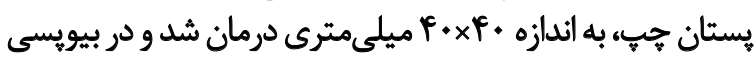

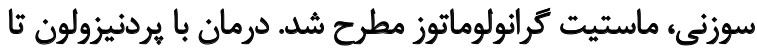
سه هفته پپس از زايمان ادامه داشت.

ماستيت كرانولوماتوز ايديوياتيك را اولينبار در سال

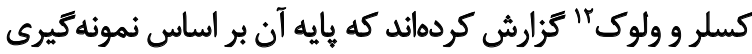

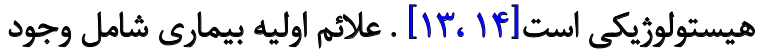

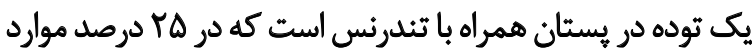

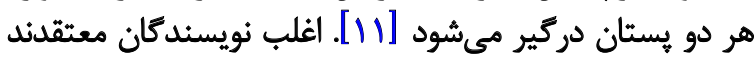

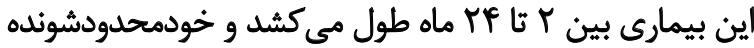

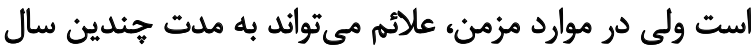

\section{Kessler \& Wolloch}

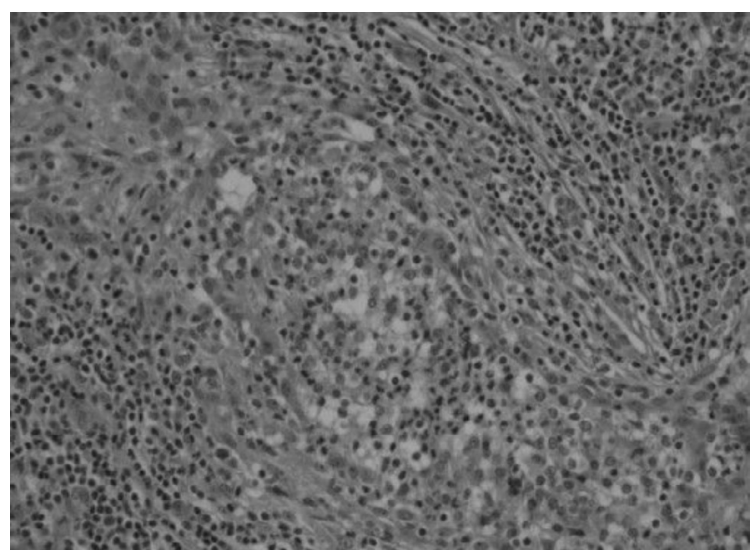

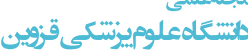

شكل ". ماستيت حاد با سلول هاى يلمىمورفوئيك؛ كرانولومهاى كاملاً مشخص

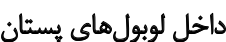

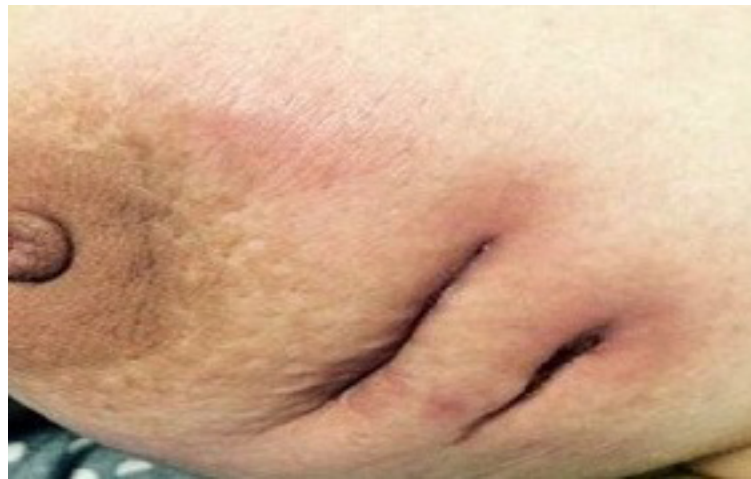

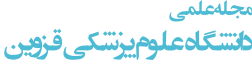

شكل ا. يستان جيب در خائم باردار مبتلا به ماستيت كرانولوماتوز باردارى

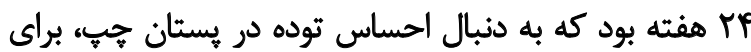

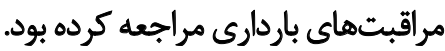

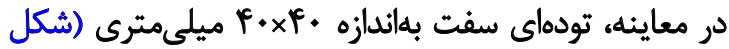

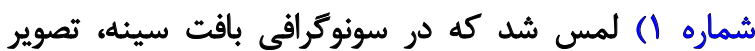

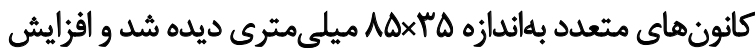

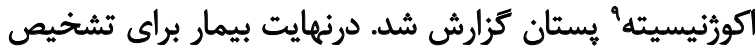

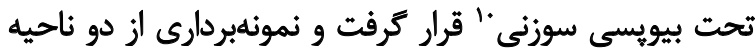

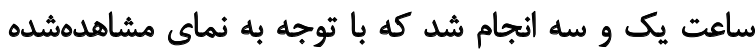

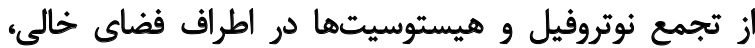

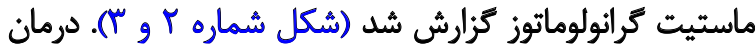

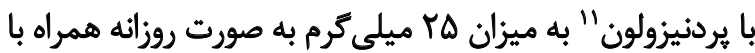

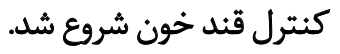

\section{Echogenicity}

10. Core niddle biopsy

11. Prednisolone

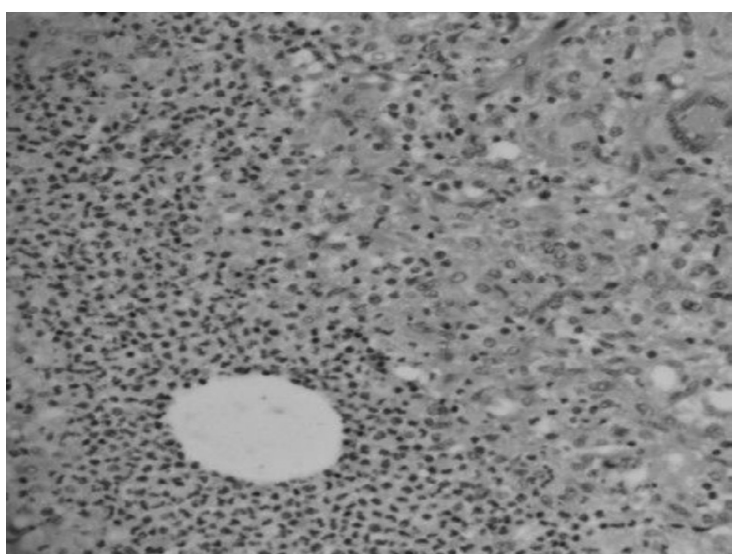

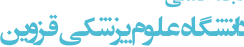

شكل †. تجمع نوتروفيل و هيستوسيثها در اطراف فضاى خالى 
اسرار نمونه انجام شد.

$$
\text { مامى مالى }
$$

حامى مالى اين يُؤهش مركز آموزشى و درمانى كوثر قزوين

$$
\text { مشاركت نويسند مَان }
$$

كردآورى اطلاعات، مشاركت در تزارش مورد و مفهومسازى:

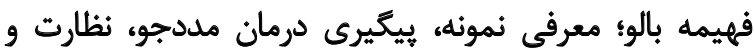

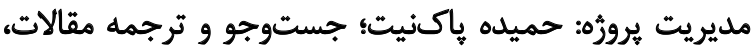

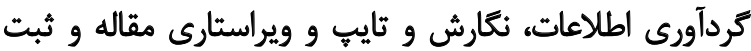

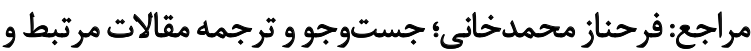

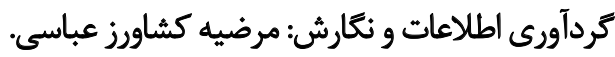

$$
\text { تعارض منافع }
$$

بنابر اظهار نويسندكان اين مقاله تعارض منافع ندارد.

$$
\text { سياسئزَارى }
$$

نويسندكان از مركز تحقيقات بالينى و كميته تحقيقات

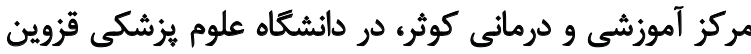
سياسكزارند إند
تداوم يابد [1ه].]

ماستيت كرانولوماتوز ايديوياتيك اغلب در زنان در سنين

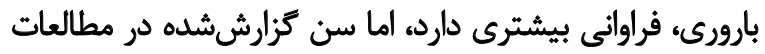

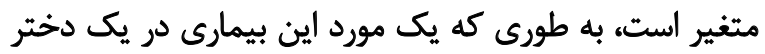

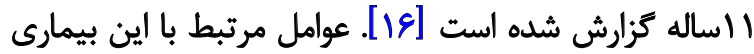

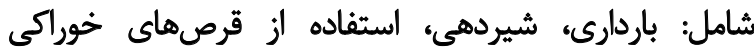

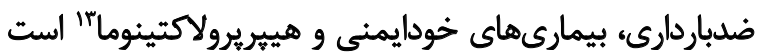

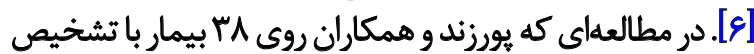

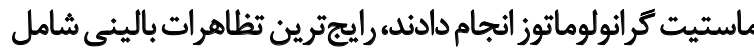

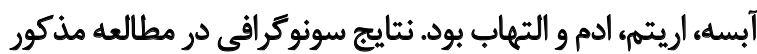

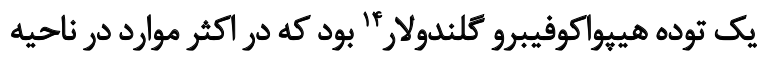

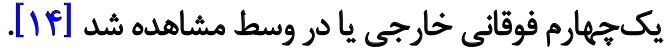
از ابزارهاى تشخيصى اين بيمارى مى توان اولتراسونوكرافى،

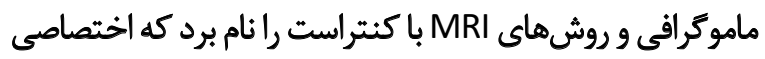

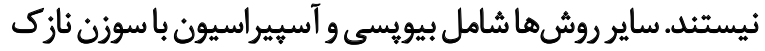

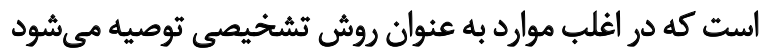
[ [I. IV] الفتبخش و همكاران براى بررسى از مداز

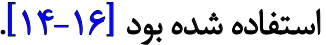

از تشخيصهاى افتراقى آن مىثتوان به بدخيمى، عفونت،

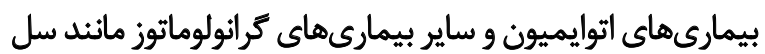

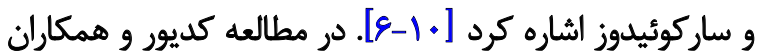

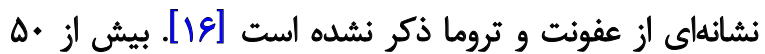

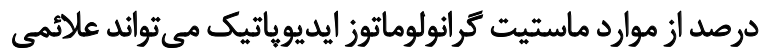

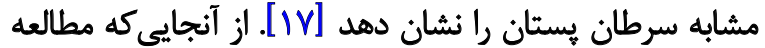

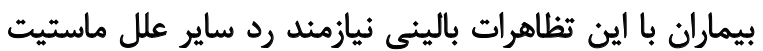

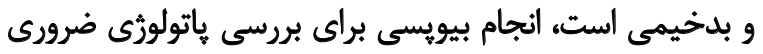

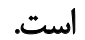

درمان يذيرفتهشده يكساني براى بيمارى وجود ندارد، ولى بورئي

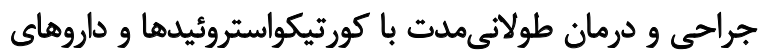

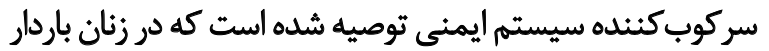

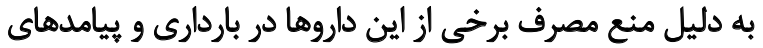

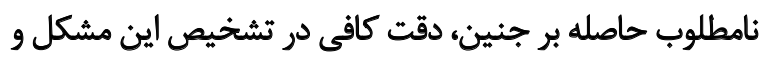

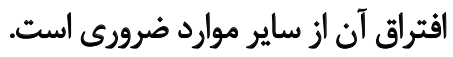

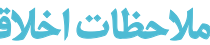

\section{بيروى أز اصول اخلاق يؤوهش}

اين مطالعه با ارائه توضيحات به بيمار و كرفتن رضايت از او

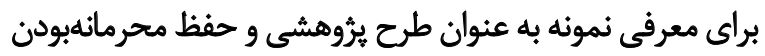




\section{References}

[1] Fazzio RT, Shah SS, Sandhu NP, Glazebrook KN. Idiopathic granulomatous mastitis: Imaging update and review. Insights Imaging. 2016; 7(4):531-9. [DOI:10.1007/s13244-016-0499-0] [PMID] [PMCID]

[2] Erhan Y, Veral A, Kara E, Özdemir N, Kapkac M, Özdedeli E, et al. A clinicopthologic study of a rare clinical entity mimicking breast carcinoma: Idiopathic granulomatous mastitis. Breast J. 2000; 9(1):52-6. [DOI:10.1054/brst.1999.0072] [PMID]

[3] Akbulut S, Yilmaz D, Bakir S. Methotrexate in the management of idiopathic granulomatous mastitis: review of 108 published cases and report of four cases. Breast J. 2011; 17(6):661-8. [DOI:10.1111/j.1524-4741.2011.01162.x] [PMID]

[4] Boufettal H, Essodegui F, Noun M, Hermas S, Samouh N. [Mastites granulomateuses idiopathiques: A propos de vingt cas (French)]. Journal de Radiologie Diagnostique et Interventionnelle. 2012; 93(7-8):620-31. [DOI:10.1016/j.jradio.2012.01.028]

[5] Diesing D, Axt-Fliedner R, Hornung D, Weiss JM, Diedrich K, Friedrich M. Granulomatous mastitis. Arch Gynecol Obstet. 2004; 269(4):233-6. [DOI:10.1007/s00404-003-0561-2] [PMID]

[6] Lin CH, Hsu CW, Tsao TY, Chou J. Idiopathic granulomatous mastitis associated with risperidone-induced hyperprolactinemia. Diagn Pathol. 2012; 7:2. [DOI:10.1186/1746-1596-7-2] [PMID] [PMCID]

[7] Taylor GB, Paviour SD, Musaad S, Jones WO, Holland DJ. A clinicopathological review of 34 cases of inflammatory breast disease showing an association between corynebacteria infection and granulomatous mastitis. Pathol. 2003; 35(2):109-19. [DOI:10.1097/01268031-200335020-00003] [PMID]

[8] Destek S. Idiopathic granulomatous mastitis: A disease mimics breast cancer appearing in pregnancy. Gen Surg: Open Access. 2018; 1(1):7-10.

[9] Larsen L, Peyvandi B, Klipfel N, Grant E, lyengar G. Granulomatous lobular mastitis: Imaging, diagnosis, and treatment. Am J Roentgenol. 2009; 193(2):574-81. [DOI:10.2214/AJR.08.1528] [PMID]

[10] Erozgen F, Ersoy YE, Akaydin M, Memmi N, Celik AS, Celebi $F$, et al. Corticosteroid treatment and timing of surgery in idiopathic granulomatous mastitis confusing with breast carcinoma. Breast Cancer Res Treat. 2010; 123(2):447-52. [DOI:10.1007/s10549-010-1041-6] [PMID]

[11] Bani-Hani KE, Yaghan RJ, Matalka II, Shatnawi NJ. Idiopathic granulomatous mastitis: time to avoid unnecessary mastectomies. Breast J. 2004; 10(4):318-22. [DOI:10.1111/j.1075122X.2004.21336.x] [PMID]

[12] Mathew M, Siwawa P, Misra S. Idiopathic granulomatous mastitis: An inflammatory breast condition with review of the literature. BMJ Case Rep. 2015; 2015:bcr2014208086. [DOI:10.1136/bcr-2014-208086] [PMID] [PMCID]

[13] Kessler E, Wolloch Y. Granulomatous mastitis: A lesion clinically simulating carcinoma. Am J Clin Pathol. 1972; 58(6):642-6. [DOI:10.1093/ajcp/58.6.642] [PMID]
[14] Pourzand A, Mohammadzadeh-Gharabaghi MA. Granulomatous lobular mastitis: A case series. J Anal Res Clin Med. 2014; 2(2):83-6. [DOI:10.5681/jarcm.2014.014]

[15] Olfatbakhsh A, Beheshtian T, Djavid GE. Granulomatous mastitis, erythema nodosum, and oligoarthritis in a pregnant woman. Breast J. 2008; 14(6):588-90 [DOI:10.1111/j.15244741.2008.00653.x] [PMID]

[16] Kadivar M, Rashidian S, Jolaii A, Dabiran S. The frequency of idiopathic granulomatous mastitis and the underlying factors in recent 5-year period. Razi J Med Sci. 2016; 23(143):18-26. [In Persian]

[17] Mizrakli T, Velidedeoglu M, Yemisen M, Mete B, Kilic F, Yilmaz $\mathrm{H}$, et al. Corticosteroid treatment in the management of idiopathic granulomatous mastitis to avoid unnecessary surgery. Surg Today. 2015; 45(4):457-65. [DOI:10.1007/s00595-0140966-5] [PMID] 
This Page Intentionally Left Blank 